\title{
Casos clínicos de esterilidad secundaria por azoospermia obstructiva subsidiaria de reparación quirúrgica
}

\author{
Balda Manzanos S*, Fernández Fernández A, Montero Rubio R, Martínez Castellanos F. \\ *Servicio de Nefrología. Servicio de Urología. Complejo Hospitalario San Millán-San Pedro. Logroño.
}

Actas Urol Esp. 2008;32(6):656-658

\section{RESUMEN}

CASOS CLÍNICOS DE ESTERILIDAD SECUNDARIA POR AZOOSPERMIA OBSTRUCTIVA SUBSIDIARIA DE REPARACIÓN QUIRÚRGICA

La esterilidad secundaria masculina es debida en una elevada proporción de casos a alteraciones en el esperma. Por otro lado, la azoospermia obstructiva constituye una causa importante de esterilidad, al ser subsidiaria de reparación quirúrgica.

Entre los factores que intervienen en la azoospermia obstructiva encontramos la cirugía tras herniorrafia, en donde cabe destacar los procesos inflamatorios subsiguientes, y la lesión epididimaria o epididimitis previa.

La importancia de los casos presentados radica actualmente, en la elevada frecuencia de dichas etiologías y en la identificación de causas obstructivas, dado que son potencialmente resolubles.

Palabras clave: Esterilidad secundaria. Azoospermia. Inflamación.

\section{ABSTRACT \\ CLINICAL CASES ABOUT SECONDARY STERILITY CAUSED BY OBSTRUCTIVE AZOOSPERMIA WITH SURGICAL REPAIR POSSIBILITIES}

Secondary sterility in male patients is due to sperm alterations in a high proportion of cases. But, obstructive azoospermia set up a very important cause of sterility, because a surgical repair is possible.

Some of the factors cause obstructive azoospermia are the herniorrhaphy intervention, including secondary inflammatory reaction, and epididymal lesion or epididymitis.

Currently, both cases are important due to high frequency of these aetiologies. Besides we should identified obstructive cause to provide a surgical repair.

Keywords: Secondary sterility. Azoospermia. Inflammation.

$\mathrm{L}^{2}$ a esterilidad secundaria es un motivo de consulta relativamente infrecuente, que merece ser estudiado con especial atención. Una historia clínica detallada orienta en un porcentaje elevado de casos, hacia el posible origen de dicha esterilidad, cuando la persona afecta es el varón.

Un motivo a tener muy en cuenta son las causas obstructivas, que han de ser investigadas de modo exhaustivo, dado que la resolución quirúrgica es factible en muchos casos.

\section{CASOS CLÍNICOS}

\section{Caso $\mathbf{n}^{\circ} \mathbf{1}$}

Paciente varón de 41 años intervenido de orquidopexia derecha en su infancia y de herniorrafia inguinal izquierda hace dos años. La técnica quirúrgica se realizó sin tener que recurrir al uso de malla de polipropileno. Padre de un niño de 5 años, consulta por esterilidad secundaria. En el seminograma se aprecia azoospermia, siendo el estudio hormonal normal. En la ecografía testicular se 
observa testículo derecho disminuido de tamaño, con un volumen testicular de $9 \mathrm{~cm}$, siendo el teste izquierdo normal.

Bajo anestesia general se realiza deferentografía con canulación microquirúrgica del conducto deferente, en la que se aprecia un stop completo (Fig. 1) a nivel del canal inguinal izquierdo. A continuación se explora quirúrgicamente el canal inguinal, identificándose un conducto deferente englobado en fibrosis secundaria a la herniorrafia, sin apreciarse sección o ligadura del mismo. Una vez resecado el trayecto estenótico, se comprobó la permeabilidad distal del mismo mediante la introducción de suero salino. Se realizó vaso-vasostomía microquirúrgica en dos planos con puntos sueltos, utilizando sutura monofilamento de Nylon de 10 ceros.

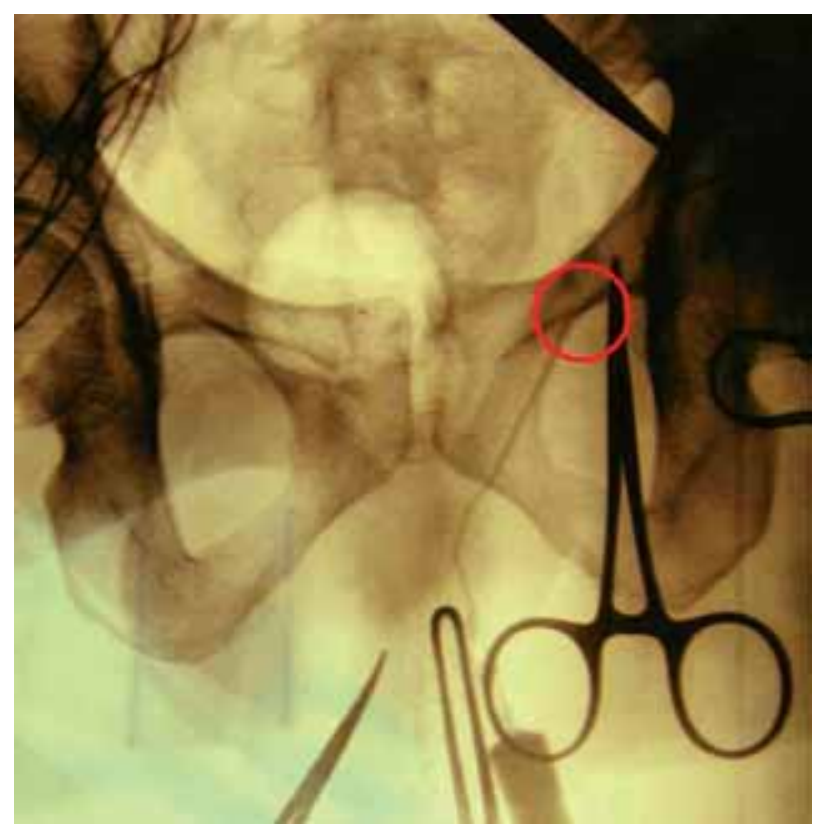

FIGURA 1. Deferentografia.

En los seminogramas de control se apreció un progresivo aumento del número de espermatozoides alcanzando los 40 millones, con una motilidad de 19\% rápida progresiva.

En la actualidad, el paciente se encuentra en programa de técnicas de reproducción asistida mediante capacitación seminal.

\section{Caso $\mathbf{n}^{\circ} 2$}

Varón de 45 años con antecedentes de herniorrafia derecha hace 10 años. Con su actual pareja es padre de un niño de 6 años. Hace cuatro años fue tratado de una epididimitis izquierda. Consulta por esterilidad secundaria de un año de evolución. En el análisis hormonal (LH, FSH, hormonas tiroideas y TSH) no se evidenció alteración. En el seminograma se apreció una oligoastenozoospermia severa $\left(\mathrm{n}^{\circ}\right.$ de espermatozoides: 1.000, con movilidad A+B del 10\%).

Con la sospecha de lesión obstructiva de la vía seminal post-epididimitis izquierda se realiza una exploración microquirúrgica, apreciándose un conducto deferente indemne así como una dilatación del túbulo epididimario en la cabeza del epidídimo. Se realiza epidídimo vasostomía latero-terminal con puntos sueltos de Nylon de 10 ceros. En los controles analíticos posteriores se identifica una mejora progresiva en el número de espermatozoides, alcanzando cifras normales en cuanto al número total a los 6 meses de la intervención, persistiendo una astenozoospermia leve.

En este caso hubo embarazo los 9-10 meses de la intervención sin precisar técnicas de reproducción asistida.

\section{DISCUSIÓN}

Las causas masculinas de esterilidad son tan frecuentes como las femeninas, oscila alrededor del $40 \%$ de todos los casos, siendo los dos hallazgos más importantes la azoospermia y la oligozoospermia $^{1}$. Un estudio inglés encontró que las alteraciones o defectos del esperma representaban el $24 \%$ de los casos, mientras que la azoospermia obstructiva sólo representaba el $2 \%{ }^{2}$.

La herniorrafia es una de las intervenciones quirúrgicas mas frecuentes y puede lesionar la vía seminal de diversas maneras ${ }^{3}$, así por ejemplo en la situación mas extrema, se puede llegar a producir una atrofia testicular por lesión vascular, que sucede en el $1 \%$ de las herniorrafias ${ }^{4}$, siendo también posibles otras formas de daño de la vía seminal como la ligadura directa del deferente, su sección, o el compromiso vascular de un segmento de deferente ${ }^{3}$. La compresión extrínseca debida a procesos inflamatorios que dan lugar a fibrosis, derivados de la cirugía constituye un factor causal de obstrucción deferencial ${ }^{5,6}$. Así lo fue en uno de nuestros casos, ya que en la exploración quirúrgica se pudo comprobar que el deferente no estaba ni seccionado, ni englobado en ligadura quirúrgica alguna. Este tipo de situaciones hace pensar que el porcentaje real de obstrucción deferencial post-herniorrafia sea mucho mas frecuente de lo que se cree. 
También se ha de señalar que la lesión post cirugía en la infancia sobre el canal inguinal, bien sea en el entorno de una herniorrafia o de una orquidopexia, es frecuente, y tal vez, más de lo pensado ${ }^{4}$, ya que su repercusión sobre la capacidad fértil, sólo se descubre cuando se lesiona el sistema de la vía seminal contra lateral.

Otro factor a tener en cuenta como causa de obstrucción de la vía seminal es la lesión epididimaria post epididimitis ${ }^{7}$. El epidídimo está constituido por un órgano flexuoso y compacto de 6-7 cm de longitud y de suma fragilidad, siendo la cola epididimaria la zona más sensible a las infecciones ${ }^{8}$; si lesionan la pared del tubo epididimario, pueden causar obstrucción y esterilidad secundaria ${ }^{9}$, y así lo fue en el segundo de nuestros casos.

La importancia de los casos que presentamos radica en dos aspectos relevantes: por un lado el hecho de que se trata de dos situaciones muy frecuentes, pues la herniorrafia es una de las intervenciones más habitualmente realizadas en un hospital $^{3}$ y la epididimitis es una de la infecciones más frecuentes de la vía seminal en el varón por debajo de los 40 años $^{7}$. Por otro lado se trata de lesiones que cursan con obstrucción de la vía seminal, y que son potencialmente resolubles mediante técnicas de reconstrucción microquirúrgica de la vía seminal ${ }^{3}$.

\section{REFERENCIAS}

1. Sciarra J. Infertility: an international health problem. Int $\mathrm{J}$ Gynecol Obstet. 1994;46(2):155-163.

2. Hull MG, Glazener CM, Kelly NJ, Conway DI, Foster PA, Hinton RA, et al. Population study of causes, treatment, and outcome of infertility. Br Med N (Clin Res Ed). 1985;291(6510):1693-1697.

3. Sheynkin YR, Hendin BN, Schlegel PN, Goldstein M. Microsurgical repair of iatrogenic injury to the vas deferens. $J$ Urol. 1998;159(1):139-141.

4. Matsuda T, Horri Y, Yoshida O. Unilateral obstruction of the vas deferens caused by childhood inguinal herniorrhaphy in male infertility patients. Fertil Steril. 1992;58(3):609-613.

5. Shin D, Lipshultz LI, Goldstein M, Barme GA, Fuchs EF, Nagler $\mathrm{HM}$ et al. Herniorrhaphy with polypropylene mesh causing inginal vasal obstruction: a presentable cause of obstructive azoospermia. Ann Surg. 2005;241(4):553-558.

6. Nagler HM, Beletete BA, Gerber E, Dinlenc CZ. Laparoscopic retrieval of retroperitoneal vas deferens in vasovasostomy for postinginal herniorrhaphy obstructive azoospermia. Fertil Steril. 2005;83(6): 1842 .

7. Kretser DM, Huidobro C, Southwick GJ, Temple-Smith PD. The role of the epididymis in human infertility. J Reprod Fertil Suppl. 1998;53:271-275.

8. Pomerol JM, Arrondo JL. Recuerdo anatomofisiológico del testículo y vía seminal. En: Ediciones científicas y técnicas, S.A. Masson S.A. Práctica andrológica. Spain,1994:3-14.

9. Pomerol JM. Azoospermia. En: Ediciones científicas y técnicas, S.A. Masson S.A. Práctica andrológica. Spain, 1994:35-42.

Correspondencia autor: Dra. S. Balda Manzanos

Servicio de Nefrología. Complejo Hosp. San Millán - San Pedro.

Piqueras, 98. La Estrella - 26006. Logroño. Tel.: 941298000

E-mail autor: sac0507@yahoo.es

Información artículo: Nota Clínica

Trabajo recibido: diciembre 2006

Trabajo aceptado: marzo 2007 